\title{
LA SOCIOLOGÍA ALEMANA \\ Y SU APORTE AL DESARROLLO \\ DE ESA DISCIPLINA
}

Marcelo Arnold C. Darío Rodríguez $\mathrm{M}$.

Las ciencias sociales son disciplinas que disponen de la capacidad de observarse a si mismas. Sus actividades, resultados y aplicaciones son parte de los sistemas que estudian y, en cuanto tales, son objeto de análisis e interpretación.

Este artículo ha sido elaborado en términos de una actividad autorreflexiva del quehacer sociológico. Se trata de una visión al interior del propio sistema científico.

En cuanto tema sociológico se abordará a la sociología alemana en dos planos: en tanto una variedad del desarrollo del pensamiento social occidental y en cuanto su expresión organizacional. Específicamente nos interesa dar cuenta del estado de desarrollo, temas centrales y centros académicos más importantes de que se dispone en la actualidad en la República Federal Alemana para la implementación y enseñanza de la sociología.

\section{IMPORTANCIA DE LA SOCIOLOGIA ALEMANA}

Al iniciar estas líneas no puede estar ausente algún tipo de justificación que ampare nuestra opción de analizar exclusivamente el curso del desarrollo del pensamiento sociológico en Alemania.

La sociología alemana está jalonada de pensadores que han influido fuertemente en el desarrollo de esa disciplina. Algunos de ellos, tales como Karl Marx (1818-1883), Georg Simmel (1858-1918) o Max Weber (1864-1920), han traspasado las fronteras disciplinarias afectando no sólo el ámbito filosófico sino además la economía, la historia, la ciencia política, la psicología y la antropología sociocultural. No menos notables, otros autores persisten en su carácter de fuente inagotable de ideas e hipótesis en el campo de la ciencias sociales; por ejemplo, Robert Michels (1876-1936), cuyo famoso enunciado acerca de la oligarquización de las orga- 
nizaciones políticas hasta el día de hoy tiene vigencia explicativa o Theodor Geiger (1891-1952), pionero de los estudios acerca de la estratificación social en sociedades complejas. No menos conocidos son los antropólogos Franz Boas (1858-1942), quien antes de emigrar a los Estados Unidos de Norteamérica estudió en Heidelberg, Bonn y Kiel y además trabajó en el Museo Etnológico de la Universidad de Berlín; Siegried Nadel (1903-1956), quien orientó parte de su construcción teórica en la dirección de los postulados weberianos o el psicólogo social Kurt Lewin (18901947), quien trabajó largos años en la Universidad de Berlín, entre tantos otros.

En la actualidad hay algunos personajes claves que están en la vanguardia del desarrollo teórico de la sociología occidental, tales como Ralf Dahrendorf (1929), Norbert Elias (1897), Jürgen Habermas (1926) o Niklas Luhmann (1927). Hay sociólogos contemporáneos que tienen fuerte impacto en nuestro ambiente, piénsese, por ejemplo, en latinoamericanistas tales como Hans-Albert Steger (1923), experto alemán en los temas de la cultura y organizaciones universitarias en Latinoamérica, o en importantes sociólogos alemanes que han permanecido algún tiempo en Chile, como es el caso de Renate Mayntz (1929) por 1965, o Peter Heintz (1920-1983) -que si bien era suizo de nacimiento, realizó sus estudios en universidades alemanas-, quien fue por cinco años, a contar de 1960, Director de la Facultad Latinoamericana de Ciencias Sociales. Cabe además mencionar a Franz Hinkelammert, quien fuera profesor del Instituto de Sociología de la Universidad Católica.

Al respecto, debe señalarse que este impacto de la sociologia alemana en nuestro medio continúa hasta nuestros días. El sociólogo alemán Norbert Lechner investiga en la Facultad Latinoamericana de Ciencias Sociales, y hay un número importante de cientistas sociales chilenos que han estudiado sociología en Alemania. Esta lista está encabezada por Luis Scherz, que inició por 1964 esta vinculación y que incentivó a muchos de sus alumnos a realizar en Alemania sus estudios de postgrado.

Debe quedar en evidencia, de este breve y preliminar recuento, la importancia que tiene para nosotros la sociología alemana hasta la actualidad.

Como veremos, la efectividad contemporánea de la sociología alemana toma sus raices en su tradición, en la historia de sus propias universidades y centros sociológicos y en la forma de cómo esta cultura sociológica es asumida en la actualidad y que, a diferencia de nuestras prácticas, se empieza a transmitir desde la educación secundaria.

II. LA TRADICIÓN SOCIOLÓGICA ALEMANA

Y SUS CENTROS UNIVERSITARIOS

Hasta mediados de siglo se reconocen en Alemania seis centros universitarios que gozaban de una gran reputación en materias sociológicas. Lo anterior explica que, 
con el inicio del siglo, se funda la Sociedad Alemana de Sociología en su carácter de institución científica. Los centros sociológicos a que aludimos son los siguientes:

- La Escuela de Berlin, asociada a la famosa Universidad Wilhelm von Humboldt. En ella enseñaron célebres sociólogos, tales como Alfred Vierkandt (1867-1953) -quien fue discípulo de los no menos célebres Friedrich Ratzel y Wilhelm Wundt-, Werner Sommbart (1863-1941) y Richard Thurnwald (1869-1953). Vierkandt fue un teórico que bordeó los límites de la filosofía y la psicología, interesado profundamente en elaborar herramientas conceptuales para la comprensión de la cultura y la sociedad; a partir de él se crean los cimientos de la moderna sociología fenomenológica. Su obra se vio interrumpida cuando el régimen nacionalsocialista le prohibió la enseñanza en la universidad. Sommbart es célebre por sus estudios históricos, políticos y económicos; especialmente conocida es su obra El Burgués (1913) y sus estudios sobre el origen del capitalismo en Europa, con ello se abre camino a la sociología histórica. Thurnwald, colaborador de Malinowski y Radcliffe-Brown, es el fundador de la versión germana de la sociología y antropología funcionalista y, a la vez, quien difunde la antropología sociocultural en Alemania. Específicamente, crea - como especialidad- una versión de Antropología social denominándola etnosociología. A partir de ésta se genera una escuela antropológica alemana, cuyo representante actual es W.E. Muhlmann (1904) en Heidelberg.

- La Escuela de Frankfurt. La línea de pensamiento que se originó en la Universidad de Frankfurt estuvo originalmente ligada a una perspectiva sociológica integradora, en la cual eran indistinguibles sus componentes históricos, etnológicos y filosóficos. Su precursor fue Franz Oppenheimer (1864-1943). De esa cuna surgió, posteriormente, la famosa "Escuela Crítica de Frankfurt" , bajo el alero de Theodor Adorno (1903-1969) y de la cual emergieron figuras claves de la sociologia de la década del sesenta: Max Horkheimer (1895-1973), quien desarrolló una teoría acerca de la sociedad, relacionando conceptos marxistas y psicoanalíticos en el marco de una sociedad capitalista en expansión; todo ello tuvo profundo eco en la denominada "Revolución de mayo del 68" que convulsionó a las universidades europeas; Erich Fromm (1900-1980), quien dirigió el Instituto Psicoanalítico de Frankfurt; Herbert Marcuse (1898-1979), quien integró la "Escuela de Frankfurt" mientras Adorno y Horkheimer trabajaron en la Universidad de Columbia (USA). Con algunas importantes modificaciones, la "Teoría crítica" de la Escuela de Frankfurt pervive en los trabajos de Jürgen Habermas (1929) y Claus Offe (1940).

- Sin duda la tradición sociológica clásica alemana por excelencia es aquella que está ligada a la Universidad de Heidelberg. De ella emergieron los más grandes de la sociología universal: Max Weber (1864-1920), Alfred Weber (1869-1958) 
y Karl Mannheim (1893-1947), este último - si bien era húngaro de nacimiento- desarrolló toda su labor intelectual que le dio renombre en esa universidad. En Heidelberg realizó sus estudios de doctorado además el conocido sociólogo estadounidense Talcott Parsons (1902-1979).

- La Escuela de Kiel. En ella desarrolló su trabajo uno de los precursores de las teorías del cambio y de la modernización: el conocido Ferdinand Toennies (1855-1936), entre otros.

- La Escuela de Colonia, cuyo punto de partida es la obra de Leopold von Wiese (1876-1969), quien da un fuerte impulso a la sociología empírica en Alemania. Posteriormente, René König (1906) impulsa la creación del Archivo Central para la Investigación Empírica en la Universidad de Colonia (1960), al mismo tiempo que impulsa el desarrollo de la metodología empírica y los estudios etnosociológicos. La sociología de la cultura también tuvo su asiento en esas aulas, especialmente por la influencia que ejerció en ellas Max Scheler (18741928).

- La Escuela de Münster, la cual bajo el impulso de Helmut Schelsky (1912-1984) se transformó - junto a Frankfurt y Colonia-en el más importante centro sociológico después de la Segunda Guerra Mundial. Por encargo del gobierno de su Estado Federal, Schelsky funda la Universidad de Bielefeld y crea en ella la única Facultad de Sociología que existe hasta el día de hoy en la República Federal Alemana. En esa misma universidad funda un Centro para la Investigación Interdisciplinaria, uno de los más importantes de Europa.

La tradición sociológica alemana puede llevar a una idea equivocada con respecto a su institucionalización en cuanto disciplina independiente. Hasta la Primera Guerra Mundial, Alemania sólo disponía de cátedras sociológicas dispersas. Después de ese conflicto, se produjo la verdadera institucionalización y autorización para entregar títulos en esa disciplina. Ese desarrollo fue interrumpido por 1933 con el advenimiento del nacionalsocialismo en las universidades primero y posteriormente en el gobierno. Después de la Segunda Guerra Mundial se reorga. nizaron o crearon centros e institutos de sociología en algunas universidades, tales como Frankfurt, Kiel, München, Colonia, Göttingen y Hamburgo. Pero la verdadera explosión de la enseñanza de la sociología en Alemania Federal ocurre paralela a la expansión de las universidades que acontece a partir de fines de la década del 50.

Gran parte de los centros sociológicos que hemos nombrado han ido perdiendo importancia y renombre, cediendo paso a patrones de organización universitaria modernos, que implican una alta movilidad espacial en el estamento de los profesores. Incluso las importantes orientaciones sociológicas que surgieron de sus salas de clases o seminarios no tienen hoy en día representantes que las mantengan. En la práctica, se ha roto la continuidad de la existencia de desarrollos teóricos ligados a centros universitarios específicos. 
Son justamente las universidades fundadas en estos últimos decenios quienes mejor han aprovechado las nuevas condiciones en las que se desenvuelven estas organizaciones. Para el caso de las ciencias sociales, se tiene los ejemplos de las universidades de Bielefeld y Konstanz, que concentran en sus recintos a una elite de profesores formados en otras universidades, los cuales se han sentido atraídos por el atractivo de mejores condiciones de trabajo y de las nuevas y modernas instalaciones con que cuentan estos centros. Ello explica, por ejemplo, que prestigiosos profesores como es el caso de Thomas Luckmann (1927) o de Niklas Luhmann (1927), por ejemplo, formen parte del Departamento de Sociologia de la Universidad de Konstanza y de la Facultad de Sociología de la Universidad de Bielefeld, respectivamente, y no de un centro tradicional como Heidelberg o Göttingen.

\section{TENDENCIAS EN LA SOCIOLOGÍA ALEMANA ACTUAL}

La sociología alemana posterior a la Segunda Guerra Mundial se caracterizó por haberse producido importantes discusiones teóricas, que buscaban la clarificación de conceptos, teorías y posturas ideológicas, para echar las bases de lo que habría de ser el modo de hacer sociología.

En los años posteriores a la guerra se recibe en Alemania la sociología norteamericana, cuya influencia es contrabalanceada por el intento de retomar las raíces de la sociología anterior al advenimiento del nacionalsocialismo. En efecto, la sociología alemana estaba muy desarrollada antes de 1933. Existía una cierta institucionalización, caracterizada por un número importante de cátedras de sociología en las diversas universidades alemanas. Después de 1945, las tendencias empiricistas importadas desde Estados Unidos se vieron enfrentadas a la tradición comprensiva, historicista y con fuerte base filosófica, propia de la sociología alemana.

Importante es el trabajo de Schelsky (1959), que -reconociendo la capacidad de la sociología norteamericana para la investigación aplicada - reclamaba la necesidad de una sociología con las bases teóricas más sólidas.

Dahrendorf (1966), por su parte, se declara insatisfecho con las posibilidades ofrecidas por la sociología norteamericarı y critica su base ideológica. Él postula la necesidad de una sociología crítica.

La discusión en torno al positivismo es otro foro que marca la época. Este significativo debate enfrenta corrientes epistemológicas en boga, que son representadas por Adorno y Popper, en un primer momento, y por Habermas y Albert, después.

Los años setenta fueron testigos de otra importante polémica. En ella se enfrentaron Habermas y Luhmann (1971) en una discusión en torno a la teoría sociológica de sistemas. Este debate fue continuado por una serie de teóricos, tales como Tjaden, Eder, Hondrich, Narr, Hejl, Runze, por citar sólo algunos (Maciejewski, 1973). 
Es posible, en consecuencia, señalar que en la sociología alemana se produce una serie de polémicas, derivadas de la necesidad de delimitación - desde la sociología- de su propio método, objeto y esquemas teóricos. En otras palabras, se trata de un proceso de reflexión autorreferente en que se constituye el sentido del quehacer sociológico. La sociología reflexiona sobre sí misma y, así, se autoconstruye, estableciendo límites respecto a otras áreas del pensamiento y conocimiento social. No pueden estar ausentes, por tanto, en este proceso, la filosofía, la epistemología, la psicología, la administración, la lingüística o la antropología. No resulta extraño - así entendido-que en el famoso debate sobre el positivismo no haya habido un solo verdadero positivista ni neopositivista (Lenk, 1979: 11). Tampoco es -en estos términos-asombroso que estas polémicas concluyan sin que se produzca un acercamiento entre las corrientes en disputa: tanto el debate sobre el positivismo, como el que se hace en torno a la teoría de sistemas - por citar los más importantes-, provocan, más que un consenso de los polemistas, el surgimiento de un problema y generan la inquietud en el ambiente sociológico por hacerse parte de la discusión. Son, en ese sentido, como reactivos, como impulsores de las vibraciones que mantienen a la sociología en constante actividad reflexiva. Un segundo efecto de estas polémicas ha sido el permitir que las tendencias en pugna revisen sus supuestos, perfilen sus temas $y$, en definitiva, reflexionen también sobre sí mismas.

Es difícil sobreestimar la importancia que las controversias aludidas han tenido en el desarrollo de la sociología alemana. Su significado se ubica en la base misma del modo alemán de hacer sociología: la ciencia se nutre de la discusión teórica y no puede haber una asimilación acrítica de teorías externas. La sociología reflexiona sobre si misma, y así, se reconstruye. La falta de autorreflexión significaría la muerte de la sociología.

Por decirlo, entonces, brevemente: el sentido de los debates no se encuentra en la busca de consenso, sino en el debate mismo.

La discusión sociológica ha ido variando conforme han cambiado las condiciones ambientales en las que la sociología se autoselecciona. Así, el problema de la postguerra era encontrar posibilidades de reflexión societal que alejaran el fantasma del totalitarismo. De ahí que se mire esperanzadamente hacia Estados Unidos y que incluso la propia tradición alemana sea recibida luego de haber sido filtrada y evaluada por sociólogos estadounidenses, como es el caso de Weber, cuyos trabajos son reconsiderados a través de Parsons.

Este período, sin embargo, no dura mucho y pronto la sociología alemana busca reencontrar sus raíces. A esto se une la crítica que se hace a la sociología estadounidense en la que no están ausentes los elementos ideológicos propios de la época de fines de la guerra fría.

Este debate va paulatinamente cediendo el paso a una discusión menos centrada en la crítica ideológica y dirigida, en cambio, más a la confrontación teórica 
y metodológica. Es así como Hondrich (1979: 134) llega a hablar de "fin de la ideología". Nos parece, sin embargo, que -como toda sobresimplificación- esta afirmación oculta más de lo que descubre. La sociología alemana está inextricablemente unida a la sociedad a lemana y los problemas de ésta constituyen los elementos de la reflexión de aquélla. En ese sentido, creemos que el problema ecológico, por ejemplo, tiene la importancia teórica e ideológica que antes pudo haber tenido la rebelión de la juventud o las secuelas de la guerra fría. La tendencia, por lo tanto -más que hacia un fin de la ideología-, parece conducir a un cambio de los intereses del debate: de la confrontación respecto a las consecuencias y supuestos políticos de las teorías, se tiende a discutir acerca de las capacidades de los esquemas teóricos de dar cuenta de las nuevas condiciones de la sociedad postindustrial, en las que no están ausentes las ideologías. Lo que cambia es el tema y, de ahí, pareciera variar el modo de hacer sociología que, sin embargo, se mantiene como autoselección y autorreflexión en una sociedad cambiante: si el problema es la ideología, la sociología es una crítica de ésta; si el problema es la mundialización de la sociedad o la contaminación, la sociología es una reflexión en torno a ello y a su propia capacidad teórica y metodológica de enfrentar esta nueva problemática.

Característico de este estado de cosas es el alto grado de complejidad que ha alcanzado el debate teórico. Paralela y correspondientemente al aumento de la complejidad de la sociedad, -tanto en términos de mundialización, como en términos de autoconsciencia, los problemas societales se han multiplicado en número, niveles e intervinculaciones- la teoría sociológica ha visto crecer su propia complejidad. La sociología, como subsistema de la sociedad, se relaciona con ésta en términos de complejidad y capacidades reductoras de esta complejidad.

La forma que ha asumido este aumento de complejidad de la teoría se ha reflejado en la forma de la construcción teórica, en la variedad de temas que pretende abarcar y en la confluencia de vertientes teóricas distintas. En efecto, las diferentes teorías, que hoy en día acaparan el interés de los sociólogos alemanes, comparten una fuerte base epistemológica y su problema central resulta ser el de la constitución de lo social; a esto se une el interés cada vez más creciente por teorías con pretensiones de universalidad, en el sentido indicado por Luhmann (1985), de teorías capaces de dar cuenta de lo social, cualquiera que sea el nivel en que se presente. En otras palabras, universalidad no quiere decir exclusividad, ya que puede haber teorías competidoras que expliquen los mismos fenómenos con perspectivas distintas.

La prevalecencia de estas teorias generales no niega la existencia de algunos intentos por construir teorías de alcance medio, tampoco puede negarse la importancia de trabajos empíricos que a través de análisis multifactoriales eluden pronunciarse sobre la teoría. Sin embargo, estas líneas de trabajo no aparecen definiendo una situación que se caracteriza más por la emergencia y el aumento de complejidad teórica que por los reduccionismos y parcializaciones de fenómenos. 
En lo que respecta a la confluencia de los afluentes teóricos que desembocan en las corrientes actuales, su origen parece encontrarse en la necesidad sentida de abarcar, desde una perspectiva integradora, la gran complejidad que implica lo social. Es asi como la teoría de la sociedad y las teorías de la evolución sociocultural se reencuentran en los trabajos de Norbert Elias, quien fuera durante largo tiempo asistente de Karl Mannheim en la Universidad de Frankfurt. De manera similar la fenomenología y la teoría de los sistemas sociales autopoiéticos se desarrolla por Luhmann y el funcionalismo se combina con el materialismo histórico y la psicología social en los trabajos de Habermas. Todo ello lleva a concluir que la tendencia actual, pareciera ser el alejamiento de las teorías parciales, en pro de teorías globales, más amplias y complejas, las que, por cierto, superan los estrechos límites disciplinarios de una sociología profesionalizada.

Esta breve reseña no ha pretendido hacer un examen exhaustivo de lo que es y representa la sociologia alemana en la actualidad, sino sólo indicar algunas de sus tendencias más visibles, señalando, al mismo tiempo, que éstas se encuentran en estrecha conexión y continuidad con el modo de hacer sociología propio de la tradición alemana. Se trata de una sociología que, ayer y hoy, se vincula fuertemente con la filosofía y en la cual el elemento comprensivo constituye su horizonte.

Quisiéramos finalizar este recuento remarcando que la sociologia alemana es una sociología consciente de sí misma en la que, por consiguiente, la autorreflexión constituye parte constitutiva. La socio logía del conocimiento, la sociología de la sociología, es central en la sociología alemana. No podría haber una sociología que -intentando explicar lo social- no percibiera la necesidad de dar cuenta de sí misma como fenómeno social. Una de las consecuencias de las teorías con pretensiones de universalidad es que también ellas constituyen parte de su objeto de estudio.

IV. LA INSTITUCIONALIZACIÓN DE

LA SOCIOLOGIA ALEMANA EN LA ACTUALIDAD

Si bien no es nuestra intención establecer comparaciones, subyace a nuestra presentación, y a la visión interesada del lector de este artículo, la posibilidad de relacionar las bases organizacionales y los estilos mediante los cuales se difunde la cultura sociológica y se forman los profesionales en esta disciplina, con eł aporte especifico que paises como Alemania han realizado para incrementar el status científico de las ciencias sociales.

Una breve descripción permitirá aquilatar, en su verdadera magnitud, lo que significa desarrollar una disciplina social en uno de los países que goza de uno de los más altos niveles de desarrollo económico en el planeta y que, a la vez, encabeza -al menos en Europa- la investigación científica de punta. Cuál es el efecto de este marco societal en la difusión y enseñanza de una ciencia social y cuáles son 
los tipos de estilos sociológicos que, a partir de ese ambiente, se sugieren, son interesantes interrogantes a las cuales indirectamente daremos respuesta.

Obviamente, en lo que sigue debemos reconocer el estado de nación dividida en que se encuentra Alemania en la actualidad, a consecuencia de la última Guerra Mundial; nuestra información, en consecuencia, se circunscribe al estado de la sociología en la actual República Federal Alemana.

En la República Federal Alemana existen, de acuerdo a informaciones oficiales, más de doscientos centros que imparten enseñanza superior; entre ellos, encontramos alrededor de sesenta universidades. En más de los dos tercios de ellas, existen carreras en las cuales, al menos, se imparte la sociologia a nivel de Diploma. Gran parte de ellas implementa también programas de postgrado. Esta sola cifra permite aproximarnos a la magnitud cuantitativa, en términos organizacionales, en la que se expresa la enseñanza de la sociología.

Lo anterior se complementa cuando se aborda el número de estudiantes. Ello no es fácil de determinar con exactitud, pues, de acuerdo a la modalidad alemana, los alumnos universitarios deben estudiar paralelamente dos carreras, una de ellas es la denominada "principal" y la otra "secundaria". Los estudios más recientes señalan que alrededor de 20.000 estudiantes cursan sociología como carrera principal (cf. Viehoff, L., 1984: 265). Esta cifra equivale a poco menos de la quinta parte del total de estudiantes en el área de las Ciencias Sociales, que incluye Derecho, Administración, Economía, etc.

Como resulta obvio de prever no todos los centros universitarios en los cuales se imparte la sociología tienen la misma envergadura. Una de las maneras de aproximarnos a este problema es relacionar tres tipos de indicadores, de los cuales se dispone de información actualizada: cantidad de personal académico y científico de que disponen, cantidad de estudiantes $y$, por último, cantidad de actividades lectivas que ofrecen en un período dado. A continuación presentaremos esta información considerando un ranking que sólo incluya los seis primeros casos.

Tabla 1

CANTIDAD DE PERSONAL ACADÉMICO Y CIENTIFICO POR UNIVERSIDAD (SOCIOLOGIA)

\begin{tabular}{lccc}
\hline Universidad & Académicos & Pers. Cientifico & Total \\
\hline Bielefeld & 29 & 49 & 78 \\
Berlin. FU & 17 & 50 & 67 \\
Frankfurt & 21 & 20 & 41 \\
Münster & 16 & 17 & 39 \\
Osnabrück & 16 & 15 & 31 \\
Kassel & 16 & 9 & 25 \\
\hline
\end{tabular}

Fuente: Daheim, H. et. al, 1987: 166 
Nuestro segundo indicador está en relación directa con el promedio de duración de los estudios. Para el caso de la obtención de un Diploma en sociología - equivale a una mezcla entre nuestras actuales Licenciaturas y el Grado de Magister- la duración efectiva para la obtención del título correspondiente es de doce semestres; no es extraño, por tanto, que muchos diplomados bordeen los treinta años ${ }^{1}$. Ello incide, por cierto, en lo que respecta al número de estudiantes (Tabla 2).

La oferta lectiva, esto es, los cursos, seminarios o talleres que se ofrecieron a los estudiantes durante el período $85 / 86$, por universidad, entregaron los siguientes datos (Tabla 3).

Tabla 2

ESTUDIANTES DE SOCIOLOGÍA POR UNIVERSIDAD

\begin{tabular}{lc}
\hline Universidad & Cantidad de estudiantes \\
\hline Berlin FU & 2.117 \\
Duisburg & 1.736 \\
Hannover & 1.674 \\
Göttingen & 1.119 \\
Bielefeld & 1.081 \\
Frankfurt & 1.006 \\
\hline
\end{tabular}

Fuente: Daheim, H. et. al., 1987: 173.
Tabla 3

OFERTA LECTIVAEN

EL ÁREA DE SOCIOLOGIAA

POR UNIVERSIDAD $(85 / 86)$

\begin{tabular}{lc}
\hline Universidad & Oferta lectiva \\
\hline Frankfurt & 336 \\
Bielefeld & 317 \\
Berlin FU & 288 \\
Osnabrück & 188 \\
Wuppertal & 171 \\
Hamburg & 149
\end{tabular}

Fuente: Daheim, H. et. al., 1987: 194.

Al relacionar estos tres indicadores, se pueden identificar con claridad los centros universitarios más importantes, en lo que respecta a la enseñanza de la sociología, específicamente las tres más grandes: la Universidad Libre de Berlín (Berlín FU), la Universidad de Bielefeld y la Universidad de Frankfurt, en ese mismo orden. Por cierto, ese ordenamiento no desmerece a centros universitarios más pequeños, algunos de los cuales entregan una enseñanza de la sociología de gran nivel y que,

Apreciamos que esta extensión de los años de estudio, más que obedecer a una excesiva rigurosidad en los exámenes, responde a la descontinuación voluntaria de éstos. Juega un importante rol aquí la oferta de trabajo que existe para el profesional egresado. En la actualidad existe una significativa cesantía de egresados universitarios. Frente a ello, el estudiante preferiría permanecer siéndolo $y$, de tal manera, seguir usufructuando sus prerrogativas sociales, especialmente en lo referido a los seguros médicos y la ayuda social a la cual tiene derecho y que se interrumpe al abandonar su status de estudiante. Los estudios indican que, hasta hace unos pocos años, los egresados de sociología tenían como principal fuente laboral de investigación y enseñanza $(29 \%)$, tareas de planificación o administración en organizaciones $(19 \%)$ e investigación en instituciones privadas $(14 \%)$ especialmente, en este último caso, en estudios de mercado. Su promedio de remuneración bordeaba, a fines de la década del 70, por los 2.700 marcos alemanes (cf. Schiebel, R. 1979). 
además, disponen entre su personal de profesores de alto prestigio; es el caso de las antes mencionadas Universidad de Colonia, Universidad de Heidelberg, Universidad de Münster y de la Universidad de Bremen y la de Erlangen.

En todo caso, los tres grandes centros de la sociología, antes detectados, no solamente disponen de grandes contingentes de alumnos y personal, los tres, en conjunto, ofrecieron durante el período analizado, más del $25 \%$ del total de cursos, seminarios o talleres en materias sociológicas para toda la República Federal Alema$\mathrm{na}^{2}$. Todo ello da cuenta del dinamismo interno con que cuentan y nos acerca a variables cualitativas que justifican su prestigio.

Una aproximación a los tipos de especialidades sociológicas que se ofrecen en las universidades alemanas lo entrega el siguiente listado: sociología de la empresa, sociología industrial, sociología de las organizaciones, sociología de las finanzas, sociologia económica, sociología política, sociología del Estado, sociología de las comunicaciones, sociologia de la literatura, sociología de la música y el arte, sociología histórica, sociología cultural, sociología de la ciencia, sociología del conocimiento, sociología de la educación, sociología médica y psiquiátrica, sociología rural, sociología de las profesiones, sociología jurídica, sociología del deporte, sociología del tiempo libre, sociología del trabajo, sociología de la familia, sociología de la juventud, sociología de la mujer, sociología del anciano, sociología de las migraciones, sociología militar, sociología del tránsito, sociología prospectiva, sociología de la comunidad, demografía, sociología matemática, sociolingüística, etnosociología, planificación social, sociología criminal, etc. (cf. Reimann, H., 1985: 41-51). Muchas de estas especialidades son absolutamente desconocidas en nuestro medio.

Al observar con más detalle las características de los centros sociológicos de Berlín, Frankfurt y Bielefeld pueden apreciarse entre éstos algunas diferencias significativas que perfilan una suerte de estilos distintos. De una manera superficial, puede indicarse que de la Universidad de Bielefeld emanan importantes desarrollos en la teoría sociológica, en cambio las dos universidades restantes están más orientadas a lo investigativo y metodológico. Una caracterización más específica de sus principales áreas de interés nos entrega el siguiente cuadro:

- Universidad Libre de Berlín: teoria sociológica, cambio social, sociología política y etnosociología.

- Universidad de Bielefeld: teoría sociológica, sociología del desarrolio, planificación social, sociología de la ciencia, sociología del derecho, sociología política y organizaciones.

2 Esta oferta lectiva se concentra, en un 60\%, en diez grandes áreas: métodos, teoría social, sociología económica, cursos introductorios a la sociología, sociologia del desarrollo, sociología de la cultura, macrosociología, sociología política y cursos sobre familia, juventud o mujeres. La cuarta parte de ellos se concentra en métodos y teoría social (cf. Daheim, H. et. al., 1987). 
- Universidad de Frankfurt: teoria de la socialización, sociología industrial, sociología política, sociologia de la educación y de las profesiones, sociología económica y metodología empírica (cf. Reimann, H. et. al., 1985: 28 9).

Por cierto estas caracterizaciones son meras aproximaciones y su durabilidad a lo largo del tiempo está sujeta a muchas contingencias. Ya hemos señalado que los sistemas universitarios modernos implican una alta movilidad en sus cuerpos académicos $y$, junto a ello, en sus áreas de interés. En ese mismo sentido, no puede sorprender que dos de estos importantes centros sociológicos se cobijan en universidades de muy reciente fundación, la Universidad Libre de Berlín poco después de la Segunda Guerra Mundial —dado que la tradicional Universidad berlinesa Wilhelm von Humboldt se ubica en la zona que corresponde a la República Democrática Alemana-, la Universidad de Bielefeld no tiene más de dos décadas de existencia. La continuidad de la tradición sociológica alemana no se enmarca, en la actualidad, con determinadas universidades. Se proyecta libremente en la cultura sociológica de su población, en sus bien provistas bibliotecas y, por cierto, en las mentes de quienes se han formado bajo el alero de esa tradición 3 .

Es conveniente señalar, que la tradición sociológica alemana no se encuentra —ni se ha encontrado nunca- restringida a universidades determinadas. Por tratarse de una actividad reflexiva, su impacto trasciende los limites de las universidades. Los sociólogos alemanes de importancia pertenecen -ellos y su obra-al patrimonio de la cultura alemana, y ello es reconocido en general. A lo anterior hay que agregar que tradicionalmente ha habido bastante movilidad de alumnos y profesores entre las diferentes universidades alemanas. Si se examina el historial académico de famosos sociólogos, como Weber, por ejemplo, será posible constatar su paso por varias universidades. Algo semejante ocurre con los estudiantes que -incluso hoy en día- realizan sus estudios en más de una universidad. Esto conduce a una vinculación tal en que alumnos pueden seguir a sus profesores y en que el prestigio de una Universidad puede variar dependiendo del prestigio de los académicos que en ella se encuentran en un momento determinado.

A pesar de la modificación que han experimentado las universidades alemanas y a pesar de la creación de nuevas universidades, con modelos modernos, la universidad humboldtiana sigue prestando muchas de sus características al sistema universitario alemán ${ }^{4}$. Los estudios de sociología -especialmente a nivel de

3 Entre ellos cabe consignar algunos chilenos que han realizado sus estudios de postgrado en el área de la sociología en la República Federal Alemana, entre otros: Fernando Alvarado (Münster), Antonio Berthelon (Bielefeld), Carlos Cousino (Erlangen), Eugenio de Solminihac (Bielefeld), Miguel Chavez (Bielefeld), Mario Durán (Bielefeld), Eduardo Lawrence (Münster), Pedro Morandé (Erlangen), Ernesto Moreno (Heilderberg), Luis Scherz (Münster), Gonzalo Undurraga (Heilderberg) y los autores de este articulo.

4 Ese modelo universitario adquiere forma organizacional cuando Wilhelm von Humboldt inspirado 
postgrado- se caracterizan por esta relación profesor-alumno que hace relativamente indiferente cuál sea la universidad en la que se estudia y muy importante quién sea el profesor con el cual se estudia. De aquí se desprende, también, la importancia de los profesores y su prestigio, frente a otros modelos de universidad en que el prestigio está más bien en relación a los Centros de Estudio.

La tradición sociológica alemana es muy rica y se mantiene vigente en la producción de los sociólogos actuales. Nuevamente figuras de prestigio -como las señaladas en estas páginas: Habermas, Luhmann, Luckmann, Elias - pasan a definir el modo de hacer sociología a nivel mundial. Los temas son variados y las perspectivas distintas, pero todos ellos se enmarcan en el camino señalado por los Weber, Simmel, Vierkandt y otros.

\section{BIBLIOGRAFÍA}

Daheim, Hansuluggen et. al. (ed.). Perspektiven der Soziologielehre: Tagung und Enquete zur Soziologielehre 1986. Leverkusen. 1987.

Dambendorf, Ralf. Sociedad y Libertad, Madrid. 1966

Hononch, Kari. Thesen zur soziologiscmen Theorie in der Bundesrepublik seit 1975. En Kölner Zeitschrift für Soziologie und Sozialpsychologie. 133-142 pp. 1979 .

LENK, HANS. Wissenschaftstheoretische Situation der Soziologie. En Kölner Zeitschrift fur Soziologie und Sozialpsychologie. 1979

Lummann, Nikias. Soziale Systeme: Grundriss ei. ner aligemeinen Theorie. Suhrkamp. 1985.

Maciejewsk: Franz (ed.). Theorie der Geselischaft oder Sozialtechnologie. Beitrage zur HabermasLuhmann Diskussion. Frankfurt. 1973.

Reinann, Hors et. al. Basale Soziologie: theoretische Modelle. Westdeutcher Verlag. 1985

SCrelsky, Helmut. Ortbestimmung der deutschen Soziologie. Dusseldorf. 1959

Schebel. Reve. Die Ausbildung- und Berufssituatin Frankfurter Diplomsoziologen. Eine empirische Untersuchung. En Soziologie. Mitteilungsblatt der Deutschen Gesellschaft für Soziologie.2. 1979 .

VEHOFF, L. Zur Entwicklung der Soziologie an den Hochschulen der Bundesrepublik Deutschland von 1960-1981. En Zeitschrift für Soziologie, pp. 264-272. 1984

en los principios del idealismo filosófico funda en 1810 la Universidad Friedrich-Wilhelm en Berlin, cuyas ideas centrales eran las de autonomia y libertad universitarias frente a los ambientes políticos y económicos. La universidad bajo ese signo pasa a ser un centro de la actividad cientifica y una comunidad de profesores y estudiantes cuya tarea es concretar esos objetivos. Este modelo se opone al francés o "napoleónico" fuertemente profesionalizante o al británico de tendencias aristocratizantes. 